الاحتياجات المائية لمحصول الحنطة القاسية (شام3) في منطقة الموصل للموسم

2010/2009

احمد أزهر ذنون العهان

مدرس مساعد/المعهد التقني /الموصل دنول

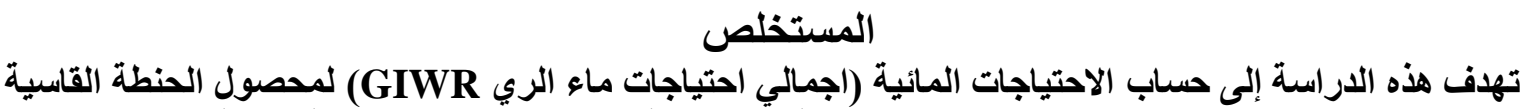

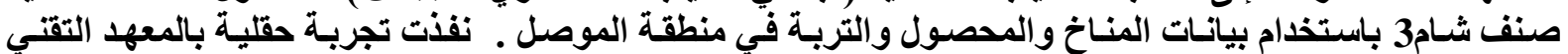

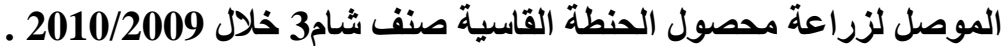

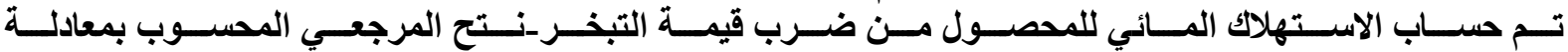

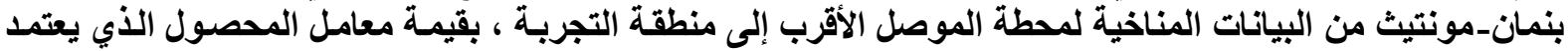

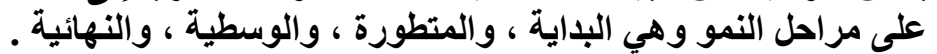

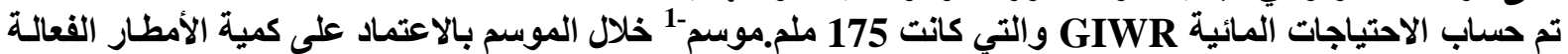

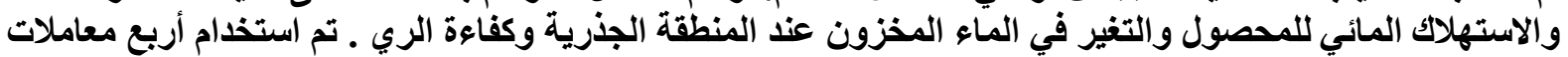

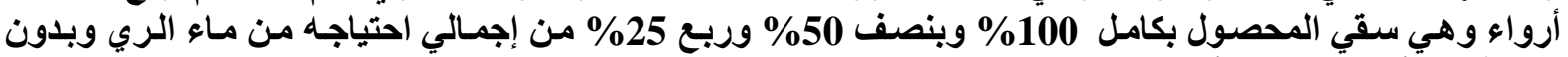

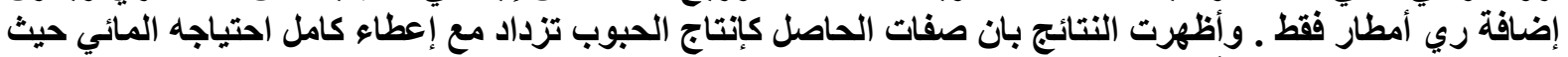

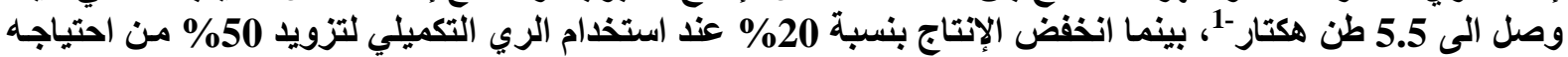

الكلمات الدالة : احتياجات ماء الري ، بنمان-مونتيث ، الحنطة القاسية ، الموصل .

\title{
Gross Irrigation Water Requirement for Durum Wheat Crop (Sham 3) At Mosul Area In Season 2009/2010
}

\author{
Ahmed Azhor Thanoon \\ Technical Institute / Mosul
}

\begin{abstract}
This study aims to determine the gross irrigation water requirement GIWR for durum wheat crop (Sham3) using climate, crop, and soil in Mosul area .

The experiment was carried out in the field of the technical institute of Mosul for the cultivation durum wheat crop cv.Sham 3 in season 2009/2010.

The consumptive water use was determined by multiplied the calculated reference crop evapotranspiration using Penman-Monteith equation based on climatological data for Mosul station which considered the nearest to the experiment, by a crop coefficient depended on the growth of stages ( initial, development , mid, and end stage) .

The GIWR was $175 \mathrm{~mm}^{-s_{e} a s o n^{-1}}$ and determined based on the effective rainfall, consumptive water use, change in soil water storage in root zone, and efficiency of irrigation . Four irrigation treatments were used $100 \%, 50 \%, 25 \%$ from GIWR , and without irrigation, only rainfall .The results showed that the crop properties such as the grain yield increases with irrigated in full GIWR and reached to 5.5 t/ha ,While it decreases by $20 \%$ with irrigated in $50 \%$ from GIWR .
\end{abstract}

Keywords: Irrigation water requirement, Penman-Monteith, Durum wheat, Mosul. 
إن الاستخدام الأمثل للموارد المائية المتاحة هو الهدف الرئيسي لتحقيق اكبر عائد اجتماعي و اقتصادي قومي دون التأثثر

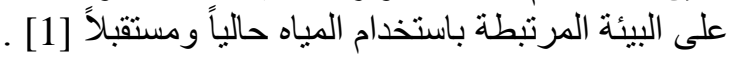

يعد محصول الحنطة من أكثر محاصيل الحبوب زر اعة في محافظة نينوى في فصل الثتاء وله مردود اقتصسادي كبير

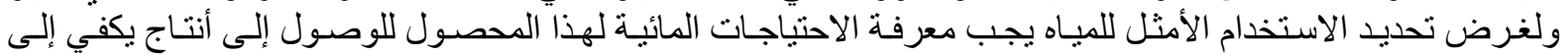
الاستهلاك المحلي و هنالك عدة مصطلحات يجب الأبن لأنعرف عليها.

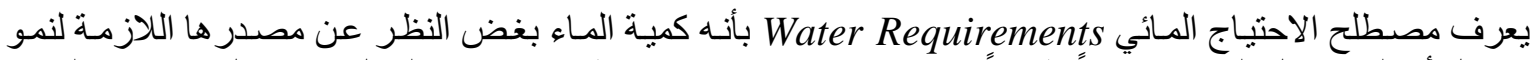

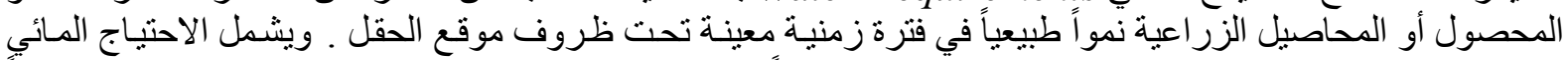

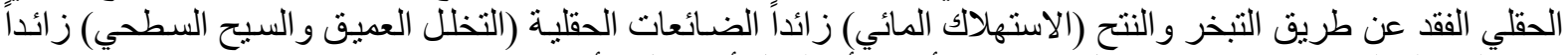

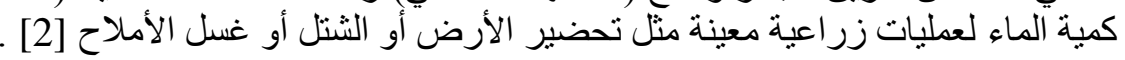

أما الاحتباج المائي للمحصول Crop Water Requirements فيعرف بأنه عمق الماء اللازم لتعويض فو فو اقد المباه

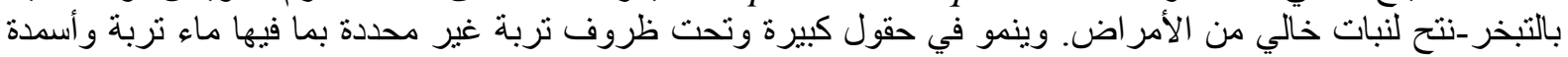
وينمو نحو الإنتاجية القصوى تحت ظروف الأبر النمو المذكورة [3ن]

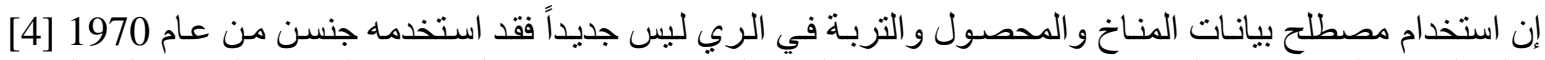

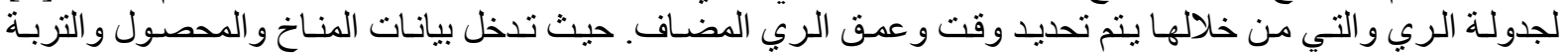
مجتمعه في حساب احتياجات ماء الري لأي محصول ينمو طبيعيا .

لتخمين التبخر -نتح لمحصول معين ، يتم عادة تخمين التبخر -نتح لنبات مرجعي من سطح قياسي ومن ثم ثن تطبيق معامل

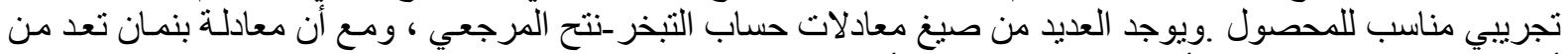

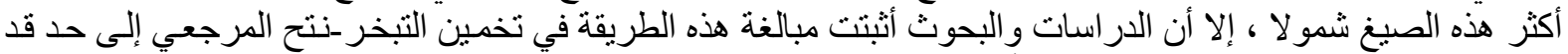

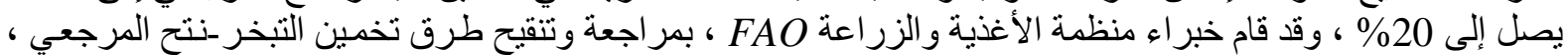

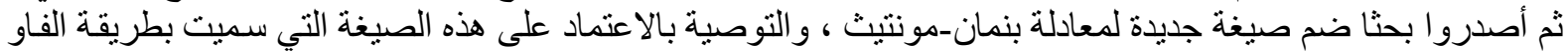

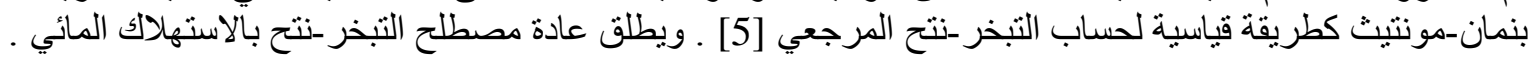

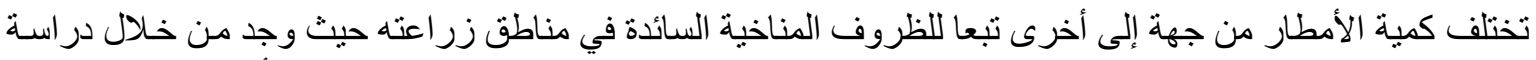

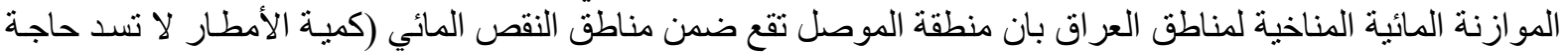
النبات من المياه بالاستهلاكك المائي) وبمقدار 340 ملم وينحصر هذا النقص في شـهري تنـرين الأول و الثناني وأثـهر آذار

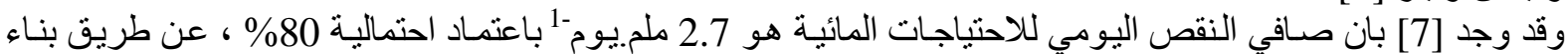

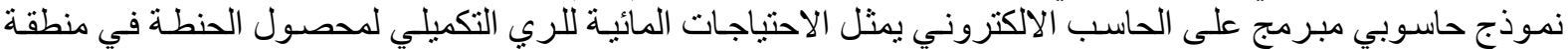

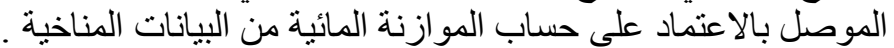

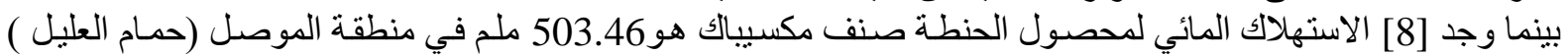

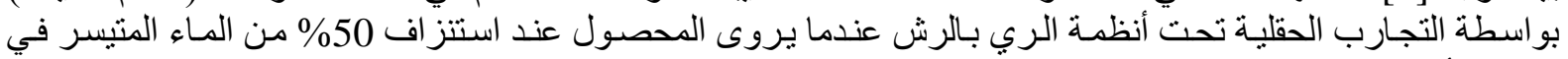
التربة ، أما احتياجات الري فكانت 256.7 ملم .

يهرف هذا البحث إلى حساب إجمالي احتياجات ماء الري لمحصول الحنطة باستخدام بيانات المنـاخ و المحصول و التربـة في تجربة حقلية في منطقة الموصل .

حساب الاستهلاك المائي لمحصول الحنطة

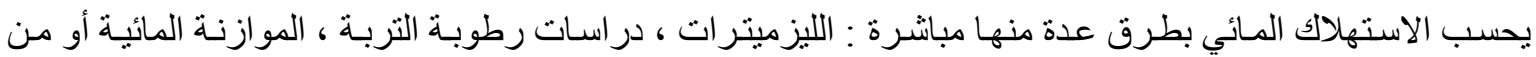

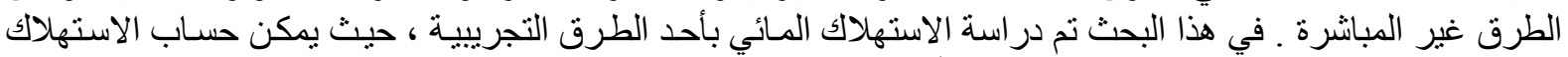
المائي لمحصول الحنطة تحت الظروف القياسية أب بدون تعطيش المحصول إلثناء نموه بالمعادلة الآتية [9] : 
$E T c=E T o * K c$

حيث أن :

ETc Kc : معامل المحصول ( بدون وحدات).

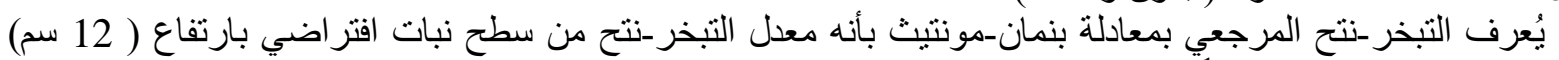

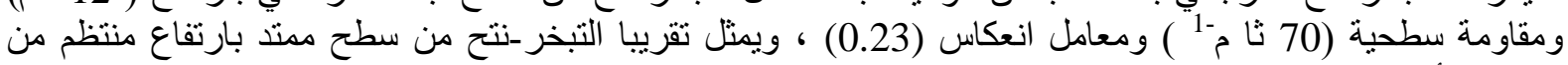

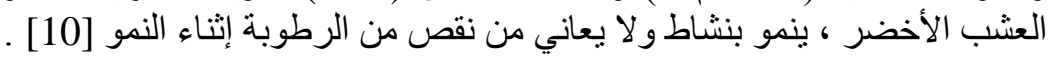

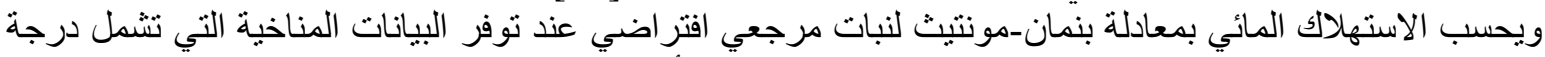

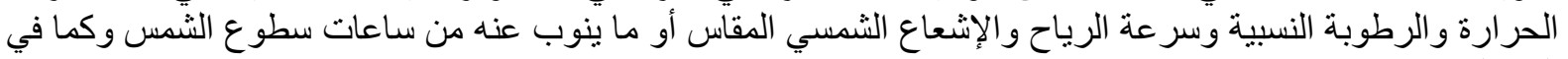

$$
E T o=\frac{0.408 \Delta(R n-G)+\gamma \frac{900}{(T+273)} U 2(e s-e a)}{\Delta+\gamma(1+0.34 U 2)}
$$

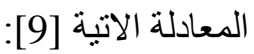

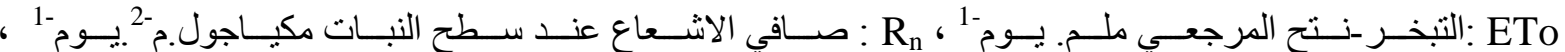

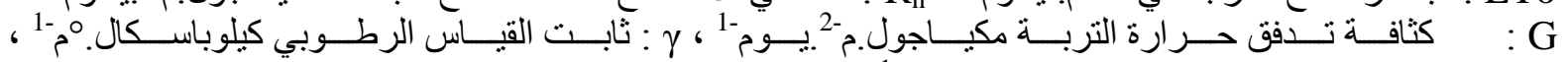

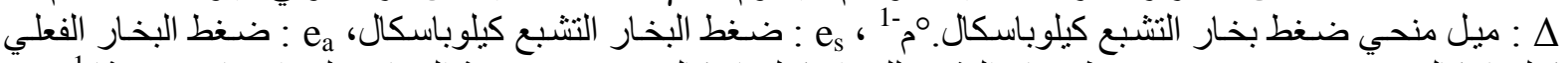

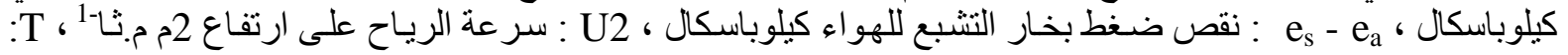

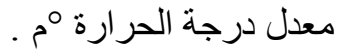
تم الاعنماد على البيانات المناخية من محطة الموصل للانواء الجوية في حساب التبخر -نتح المرجعي [11] و التي تقع

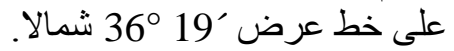

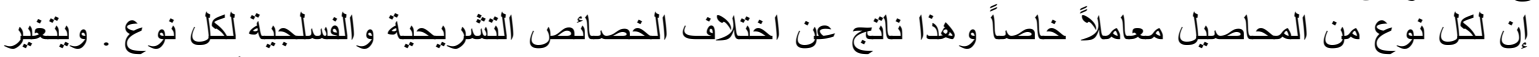

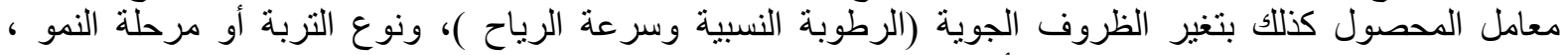

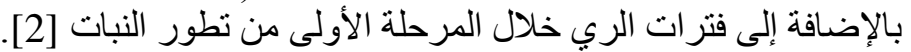

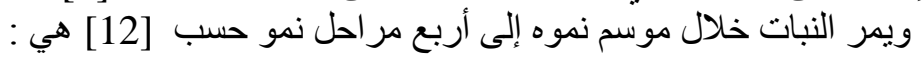

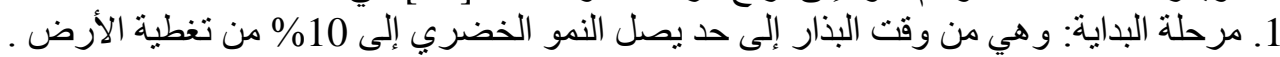

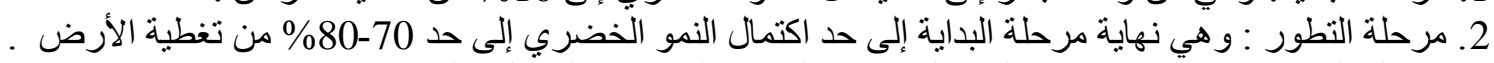

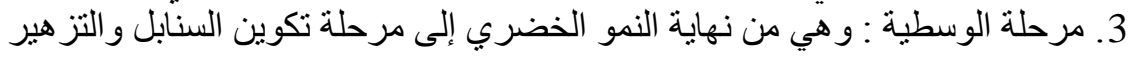

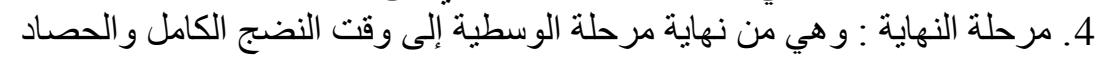

أما معامل المحصول للمر احل الأربعة فكان 0.35 ، 0.75 ، 1.15 ، 0.45 المالب 0.45 حسب [12] ـ وتستخدم طريقة التداخل بين المر احل Overlap procedure في حساب معامل المحصول على أساس شهري.

\section{طريقة العمل}

تم زر اعة محصول الحنطة صنف شام 3 ( المنشا منظمة ايكاردا في سوريا ) الذي يعتبر من أصناف الحنطة القاسية في

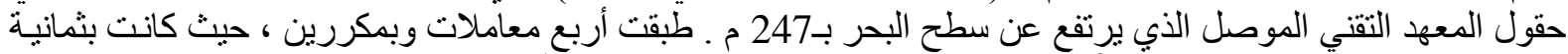

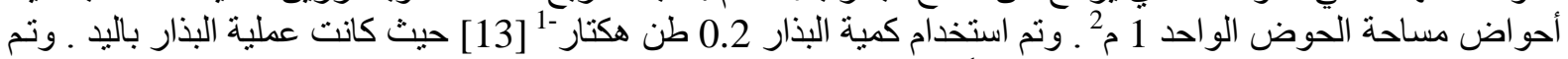

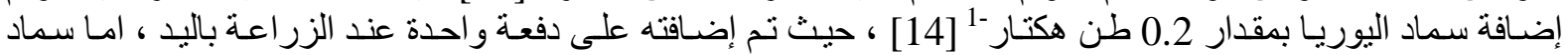

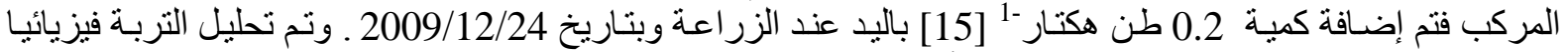

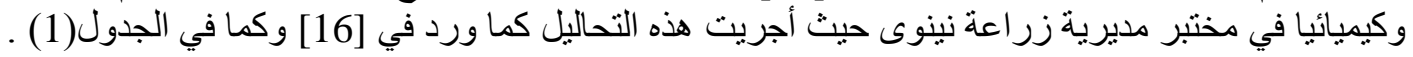

الجدول (1): التحليل الفيزيائي و الكيميائي للتربة الحقل

\begin{tabular}{|c|c|c|c|c|c|c|c|c|}
\hline \% الجبس & $\begin{array}{c}\text { البوتاسيوم } \\
\text { ppm }\end{array}$ & الفسفور & ن النتروجي & النسجة & نسبه & $\begin{array}{c}\text { الغرين } \\
\text { \% }\end{array}$ & الطين & العمق \\
\hline 0 & 193.72 & 0.7 & 70 & مزيجية غرينية & 37.65 & 47.425 & 14.925 & 15-0 سم \\
\hline 0 & 263.42 & 0.1 & 49 & مزيجية طينية & 22.575 & 42.425 & 35 & 30-15 سم \\
\hline
\end{tabular}


الاحتياج المائي

$$
I W R=\frac{E T c-P e-G W-\Delta S}{1-L R}
$$

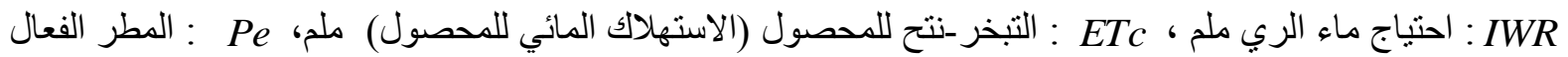

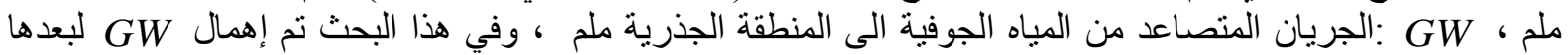

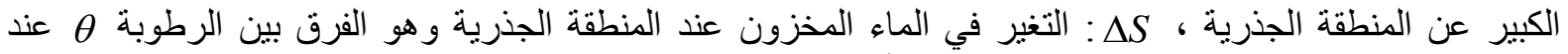

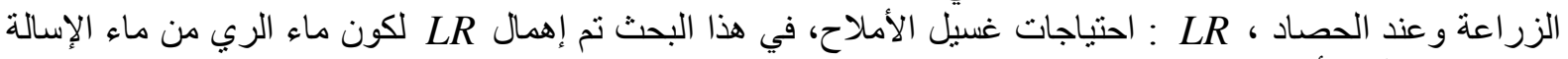

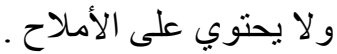

$I W R=E T c-P e-\Delta S$ وبالتالي يمكن كتابة احتياجات ماء الري معادلة رقم (3) بالشكل الأتي :

$$
\begin{aligned}
& P e=a P_{t o t} \\
& P T C
\end{aligned}
$$$$
\text { ويحسب المطر الفعال Pe بمعادلة النسبة الثابتة وهي [18] : }
$$

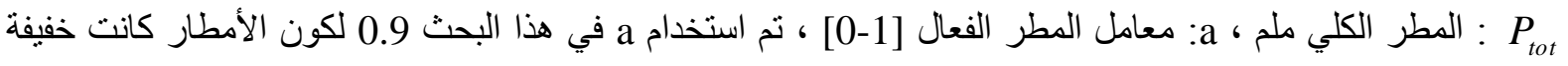

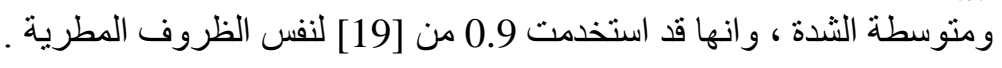

$\Delta S=\left(\theta_{a}-\theta_{b}\right) d$ ويحسب التغير في الماء المخزون $\Delta S$ على أساس شهري بالمعادلة التالية .

胃a حيث ان : عمق :المحتوب :

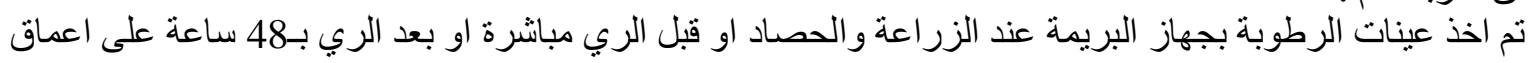

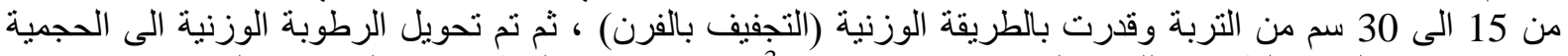

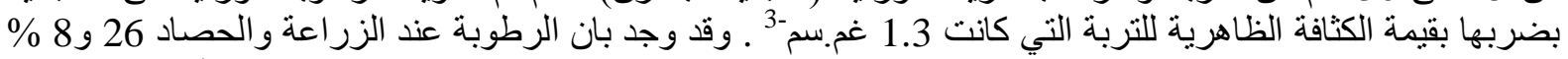

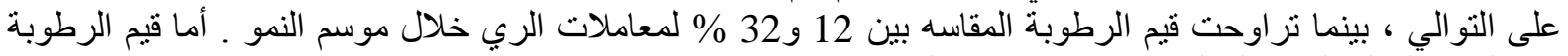

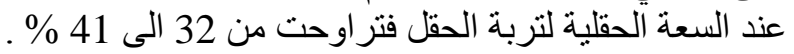

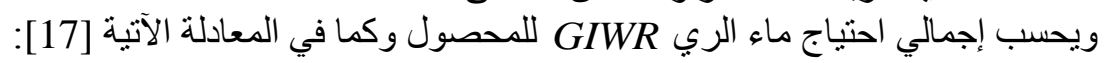

$G I W R=I W R / E f f$

GIWR

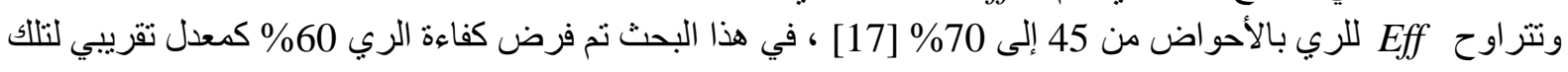
القيم . وفي حالة كون الاستهلاك المائي اقل من المطر الفعال فان IWR تكون صفر ، ويحسب الاستهلاك المائي والمطر

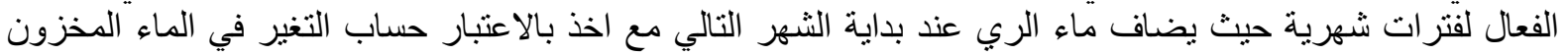

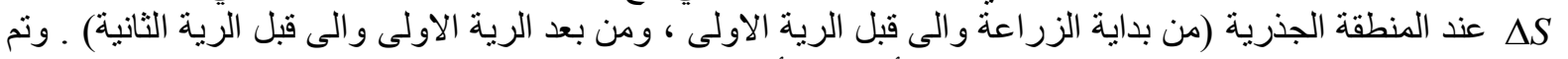

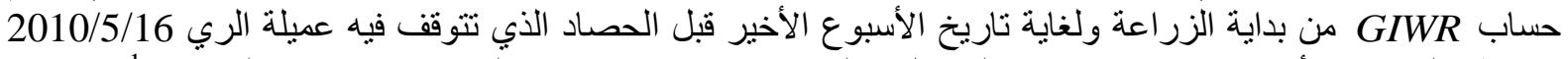

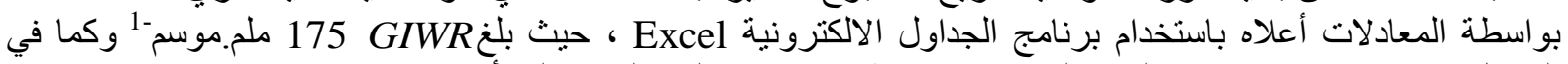

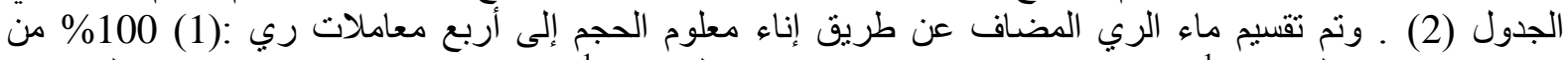

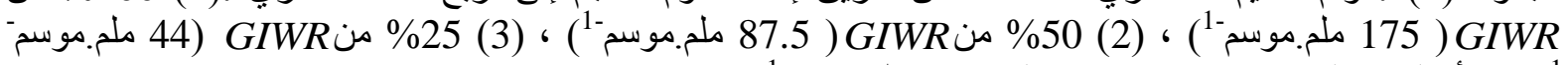

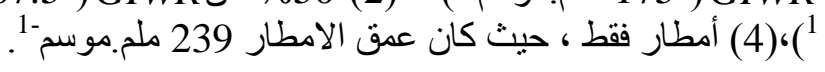


ذنون: الاحتياجات المائية لمحصول الحنطة القاسية (شام3) في منطقة الموصل للموسم 2010/2009

الجدول(2) : طريقة حساب اجمالي احتباج ماء الري إئي

\begin{tabular}{|c|c|c|c|c|c|}
\hline الرمالي احتياج ماء & الزياج ماء & المخزون المير في & $\begin{array}{c}\text { المطر الفعال } \\
\text { Pe }_{\text {e }} \\
\text { ملم }\end{array}$ & التبخر-نتح & الاشهر \\
\hline 0 & 0 & - & 51.8 & 2.3 & $\begin{array}{c}24-\text { كانون الاول } 2009 \\
24\end{array}$ \\
\hline 0 & 0 & - & 48.9 & 20 & 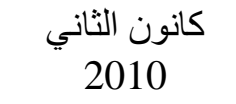 \\
\hline 0 & 0 & - & 43.3 & 43 & شباط \\
\hline 80 & 48 & 21.7 & 17.3 & 87 & اذار \\
\hline 95 & 57 & 9 & 20.7 & 86.7 & نيسان \\
\hline- & - & - & 33 & 49 & 23- ايار \\
\hline 175 & 105 & & 215 & 288 & المجموع \\
\hline
\end{tabular}

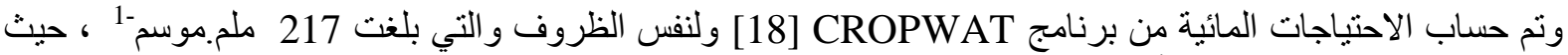

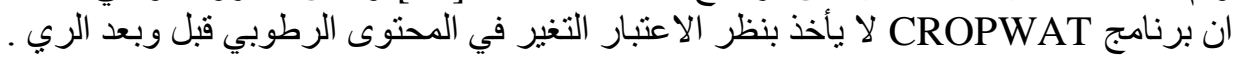

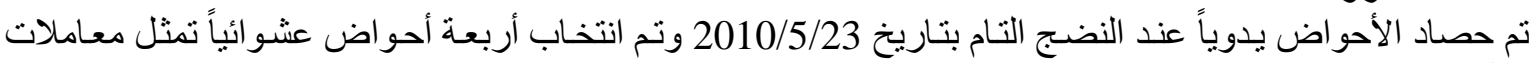

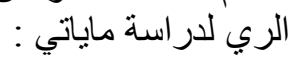

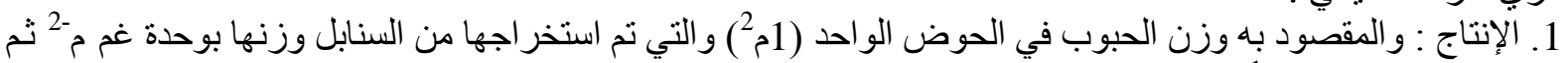
تحويله إلىى( طن هكتار -1 1 ) .

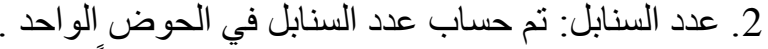

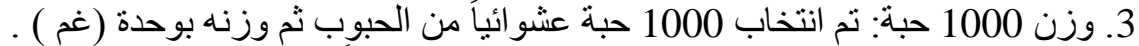
4. ارتفاع النبات: تم حساب معدل طول خمسة نباتات عشو ائياً من الحوض ون ونم القياس بوحدة ( سم ) .

كفاعة استخدام المياه

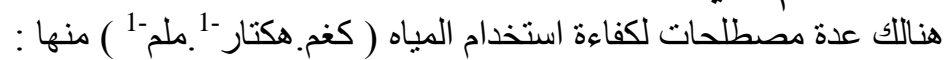

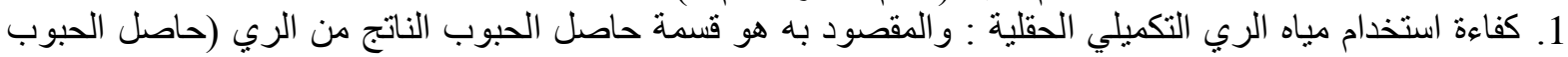

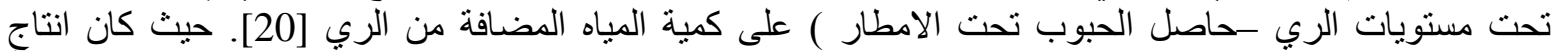

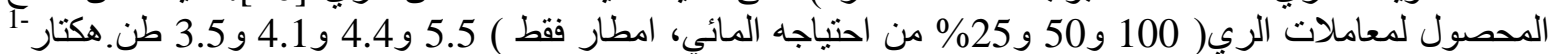

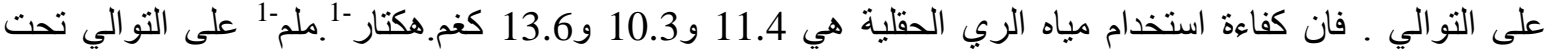
اعماق الري 175 و و87.5 و و44 ملم .

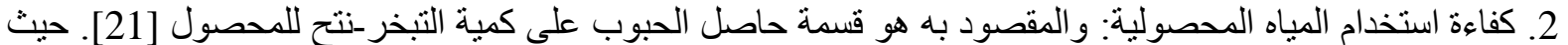

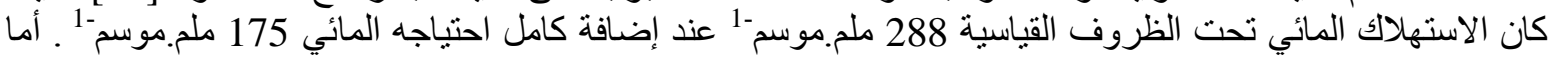

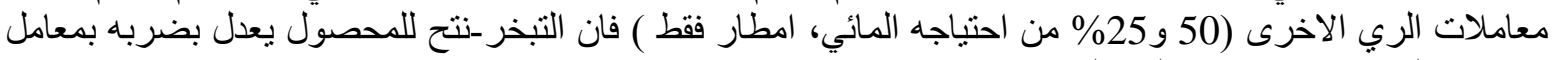

$E T c_{a d j}=E T c^{*} K s$

جهد ماء التربةٌ Ks وكما في المعادلة الاتية [9] :

ETc adj ويحسب معامل جهد ماء التربة Ks من المعادلة الاتية [9] :

$K s=\frac{T A W-D r}{T A W-R A W}$ 


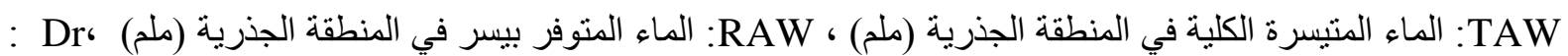

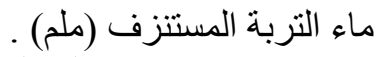

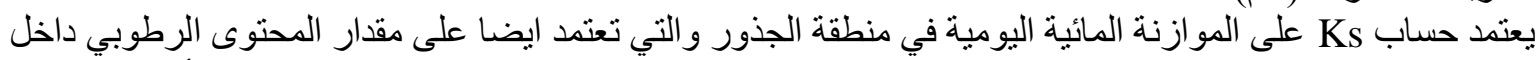

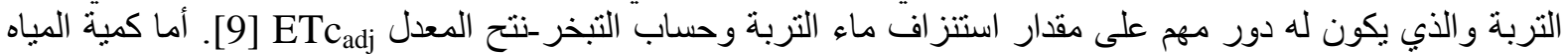

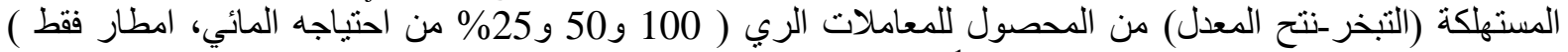
فكانت 288 ، 260 ، 267 ، 245 ، 215 ملم. 216. 19.1 ، 16.9 ، 16.7 ، 168.2 ، 16.2 كغ. هكتار -1. ملم-1 على التو الي .

نسبة النقص بالانتاج

$\left(1-\frac{y_{a}}{y_{m}}\right)=K y\left(1-\frac{E T c_{a d j}}{E T c}\right)$

يتم حساب نسبة النقص بالانتاج من المعادلة الاتية [9] :

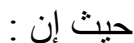

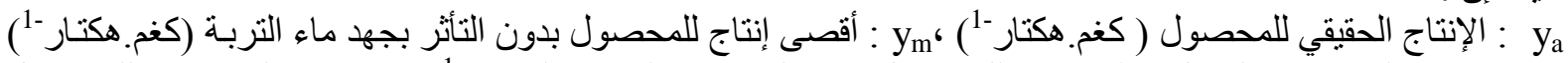

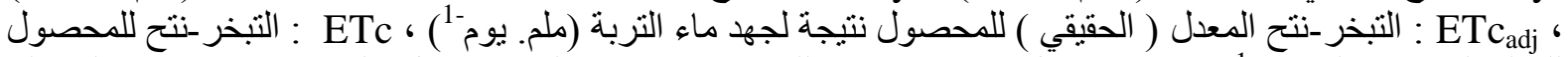

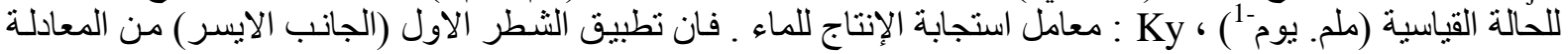

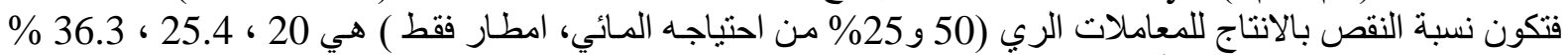
على التو الي مقارنة مع معاملة أعطاء كامل 100\% من الري من احتياجه المائي .

\section{الأنتائج والمناقشة الاحتياج المائي}

يبين الثكل (1) الاحتياج المائي لمحصول الحنطة خلال موسم النمو ، حيث بلغ مجموع الاستهلاك المائي للمحصول

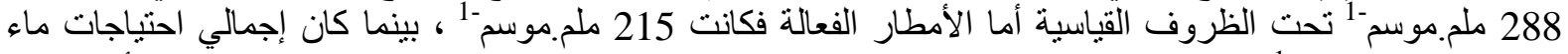

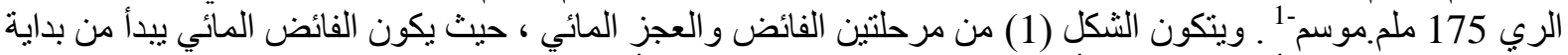

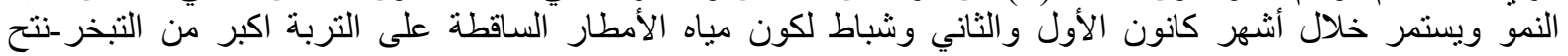

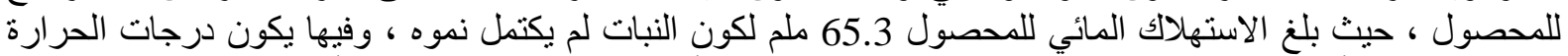

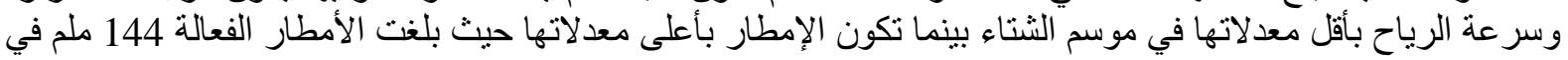

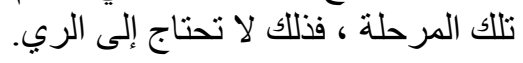

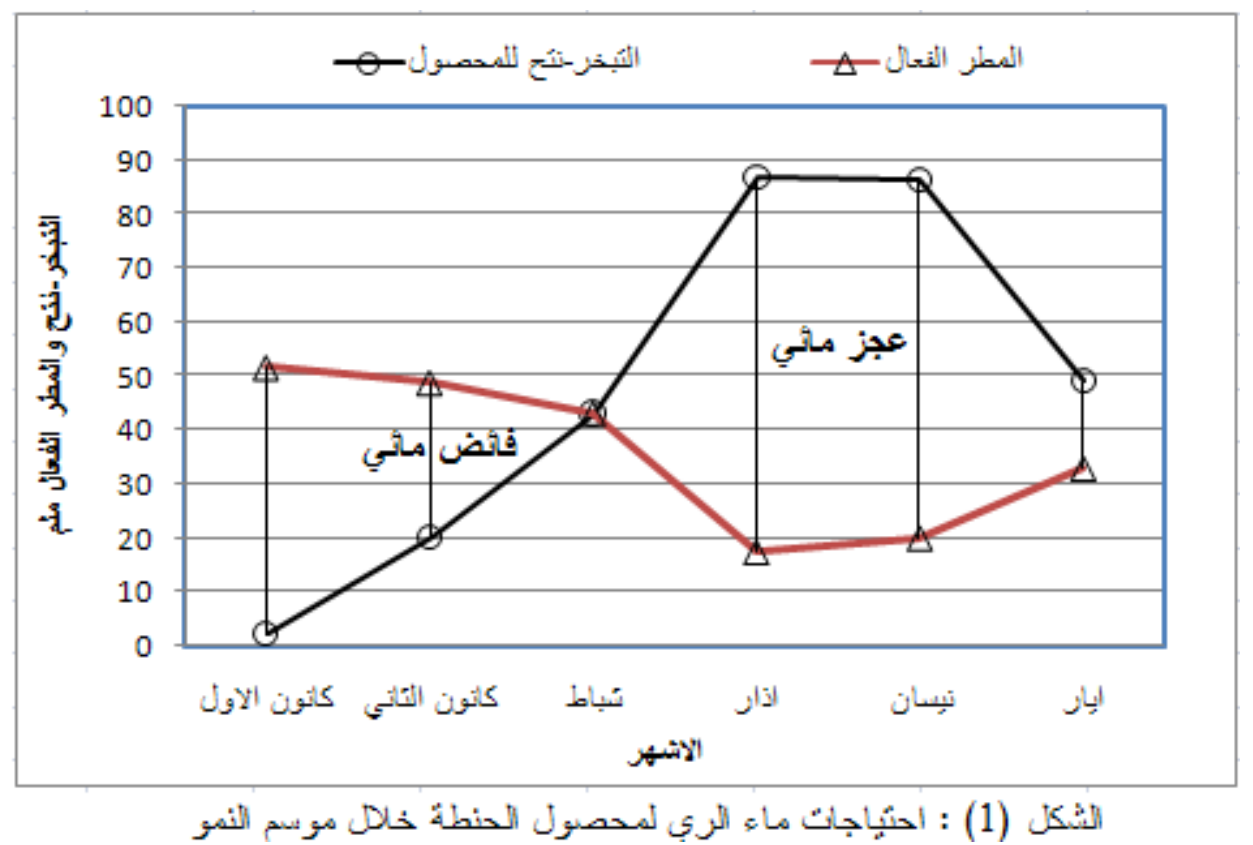




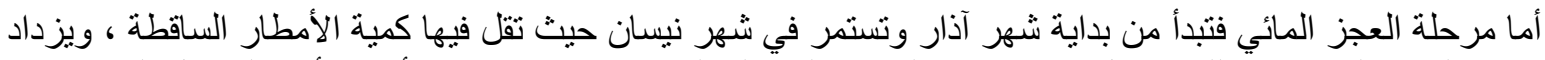

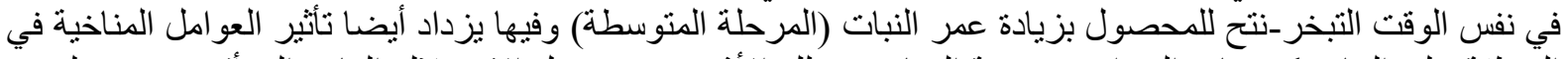

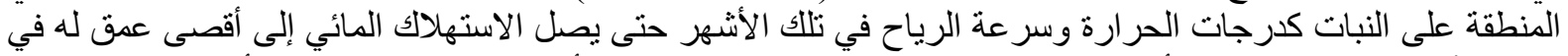

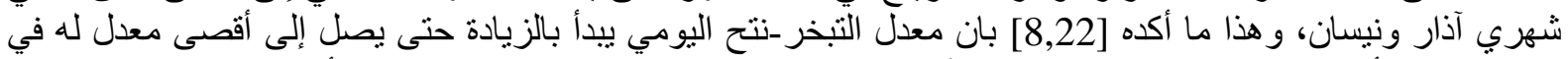

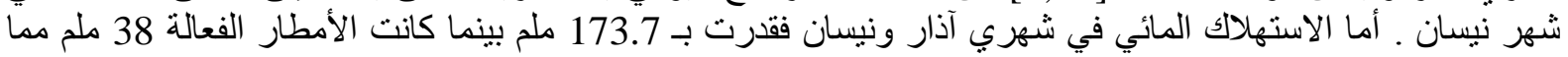

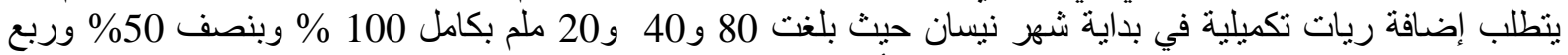

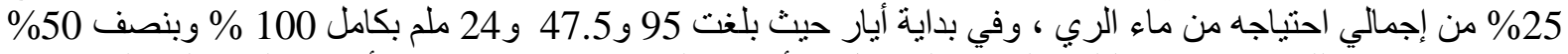

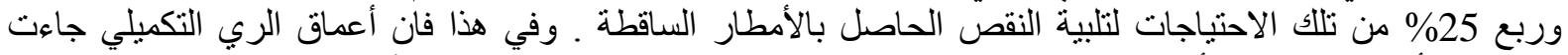

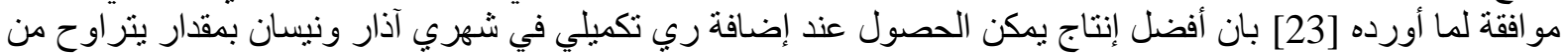

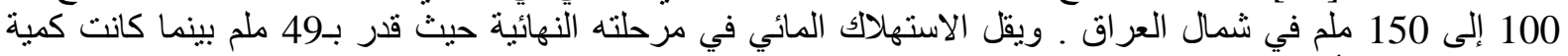
المطر الفعال في أيار 33 ملم .

\section{تأثير نسبة احتياجات الري على صفات المحصول}

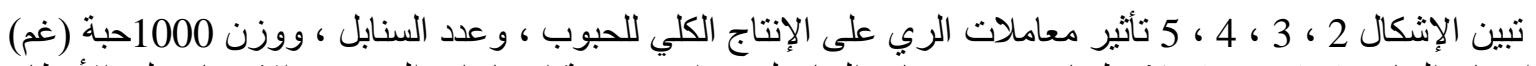

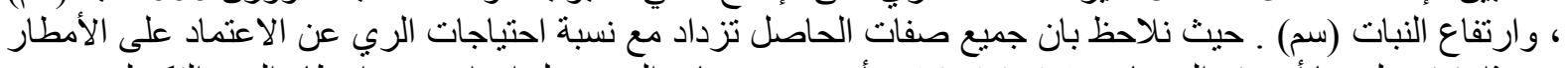

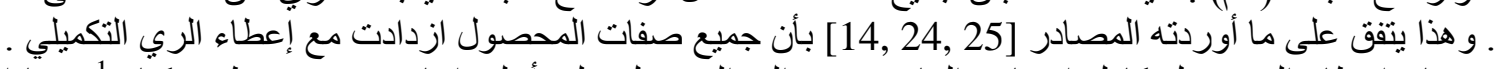

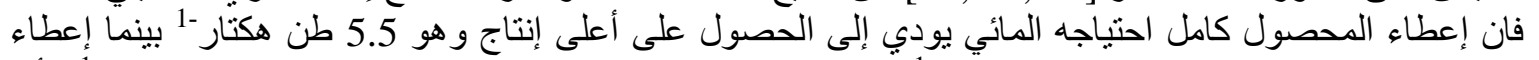

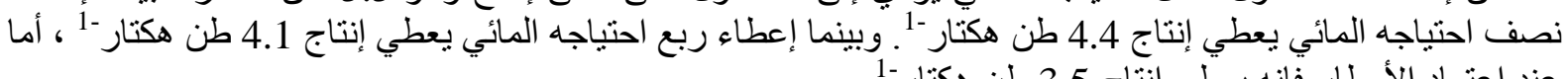

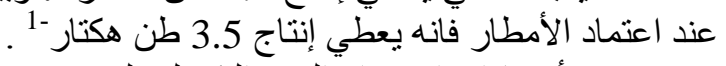

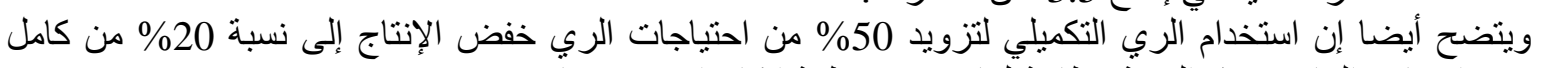

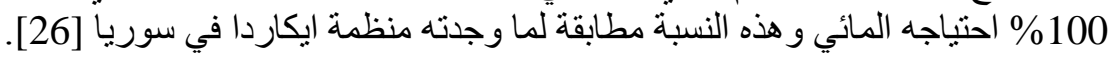
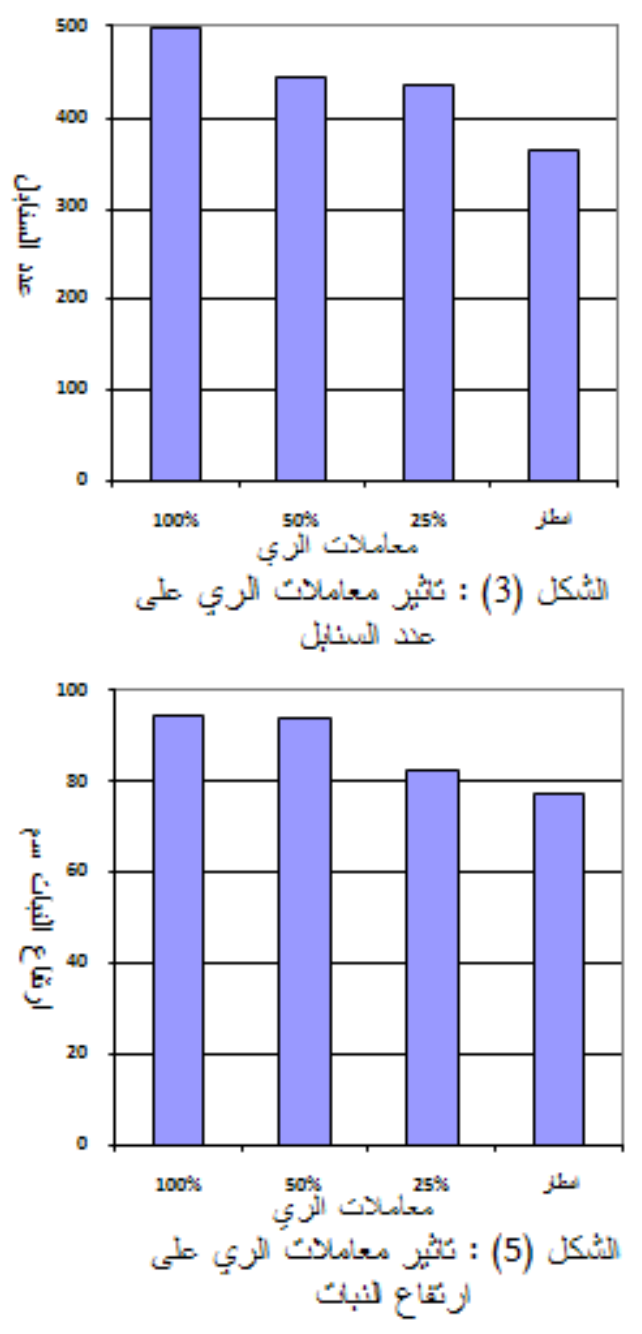
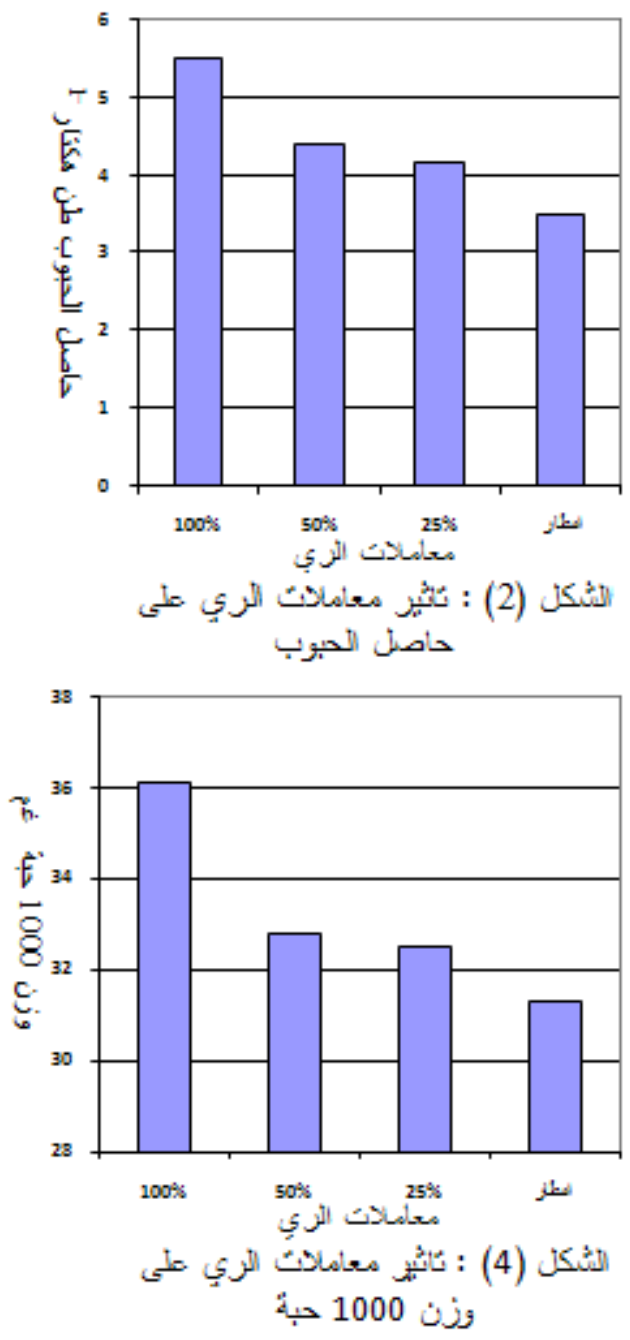
الاستتناجات و التوصيات

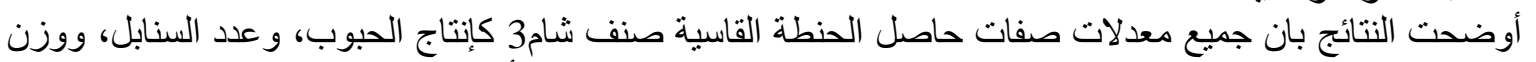

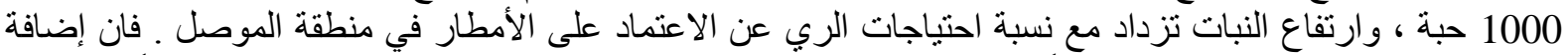

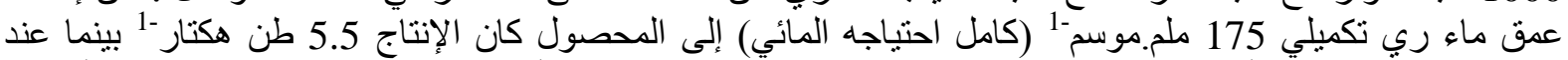

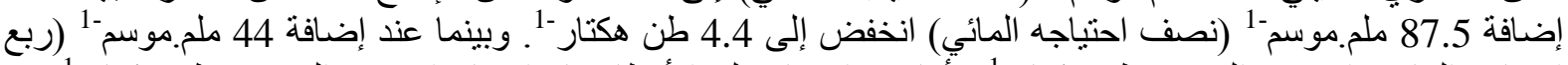
احتياجه المائي) انخفض إلى 87.1 طن هكتار -1 ، أما عند اعتماد على الأمطار فان إلانتاج انخفض إلى 3.5 طن هن هكتار -1

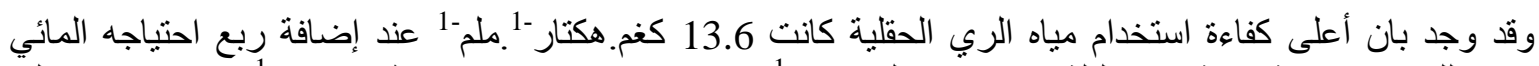

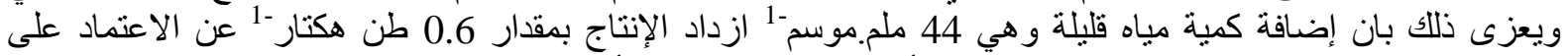

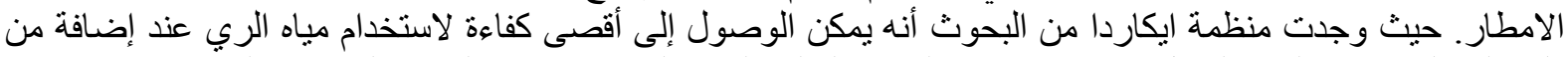

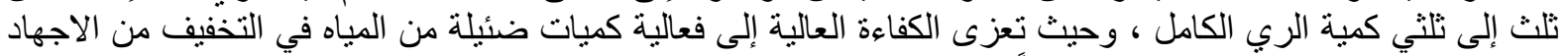

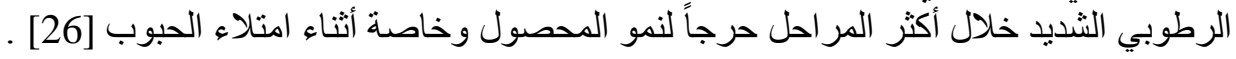

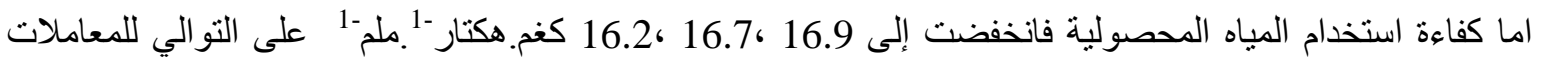

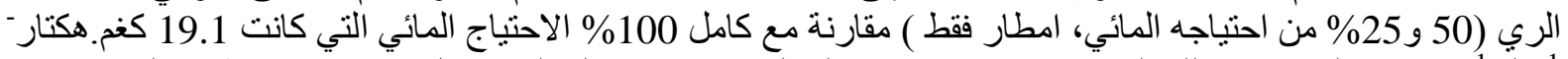

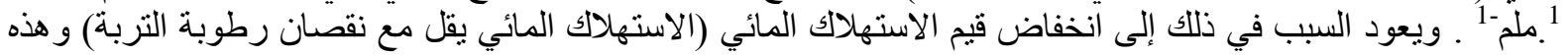

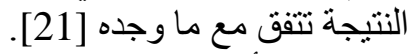

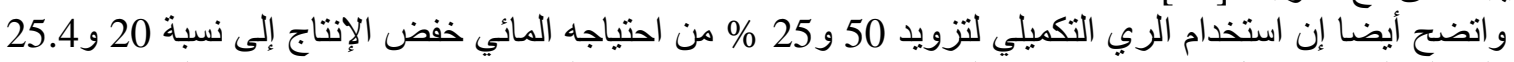

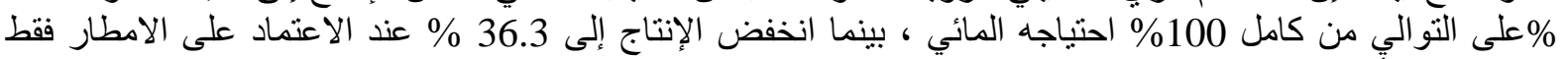

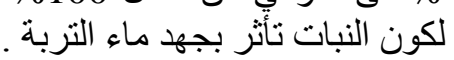

ولهذا أوصي بماياتي:

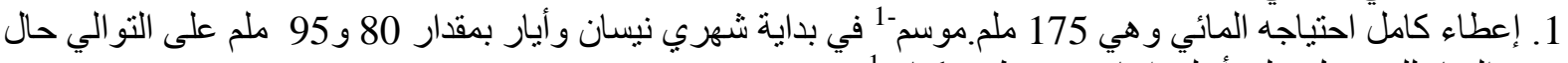

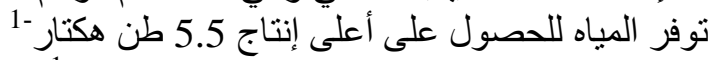

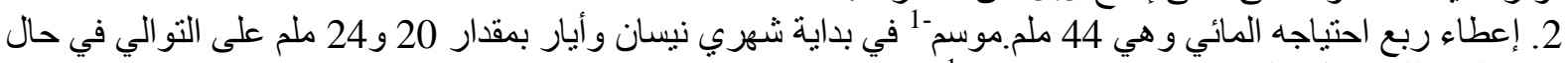

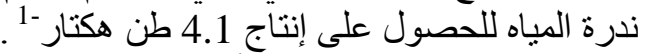
3. تكرار التجربة لعدة سنو ات أخرى.

المصادر

1. عبد الوهاب ، عبد الصبور احمد .1998. استخدام مباه الري في الأراضي الزر اعية بمصر بين الواقع والمأمول. مجلة

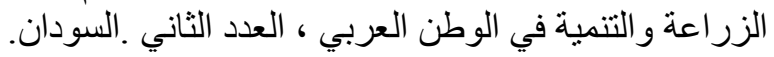
2. إسماعيل ، ليث خليل .1988. الري لئ والبز البزل. كتاب صادر عن وزارة التعليم العالي والبحث العلمي جامعة الموصل ، العراق.

3. Doorenbos, J., and Pruitt, W. O. 1977.Crop Water Requirements. FAO Irrigation and Drainage Paper 24 , Rome, Italy.

4. Jensen, M.E., Robb, D.C.N. and Franzoy, C.E. 1970. Scheduling irrigations using climatecrop-soil data. Journal of Irrigation and Drainage. Div. (ASCE) 96(IR1):25-38.

5. الجبوري ، شفيقة سلطان عبد الله حسين .2002 ـ تقيم معادلة الفاو بينمان-مونتيث لحساب الاستهلاك المائي المائي المرجعي

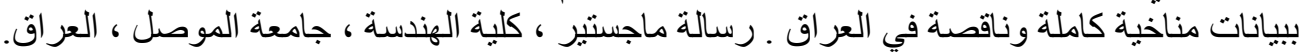

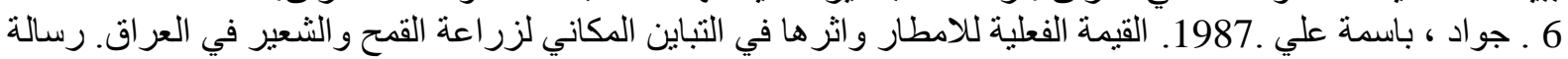
ماجستير ، كلية الاداب ، جالمعة ، جامة البصرة ، العراق.

7. Sheet E.H. 1987. Modeling supplemental irrigation water requirement for wheat crop at Mosul area. M.Sc. Irrigation Sci., Engineering college , University of Mosul, Iraq.

8. الزوبع ، محمد صالح هلوش. 1978. الاحتياج المائي للحنطة وتأثير الري التكميلي بالرش على الإنتاج ـ رسالة

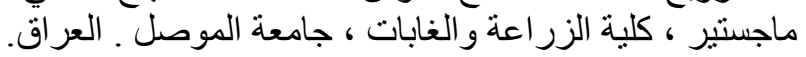

9. Allen R.G., Pereira L.S., Raes D., and Smith M., 1998.Crop evapotranspiration. Guidelines for computing crop water requirements. FAO Irrigation and Drainage Paper 56, Rome, Italy. 10. Smith, M., Allen, R., Monteith, J.L., Pereira, L.S., Perrier, A. and Segeren, A. 1992. 
Report on the Expert Consultation for the Revision of FAO methodologies for crop water requirements. FAO-AGL, Rome, Italy.

$$
\text { 11. وزارة النقل والموصلات ، هيئة الانو اء الجوية ، شعبة المناخ ، بيانات غير منشورة . }
$$

12. Brouwer , C. , and Heibloem . M. 1986. Irrigation Water Management: Irrigation water needs. FAO Training manual No.3. Rome, Italy.

13. Taghouti , M. , Gaboun, F. , Nsarellah, N., Rhrib, R., El-Haila , M., Kamar , M., AbbadAndaloussi , F. and S. M. Udupa .2010. Genotype x Environment interaction for quality traits in durum wheat cultivars adapted to different environments . African Journal of Biotechnology Vol. 9(21), pp. 3054-3062 .

14. علي ، وسام محمود .2009. تقدير الاستهلاك المائي لمحصول الحنطة (صنف أبو غريب) بطرق مختلفة تحت

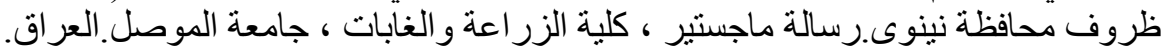

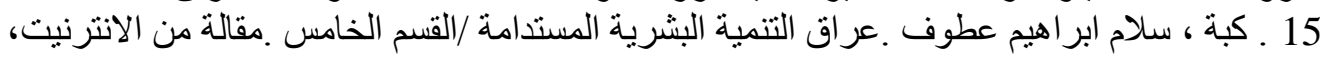
.http//www.babil-nl.org/ga51x024.htm

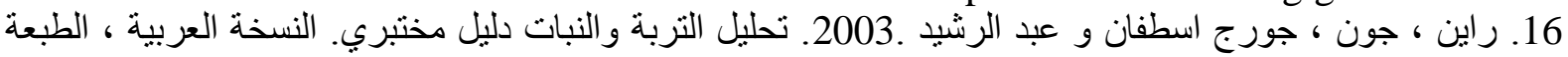

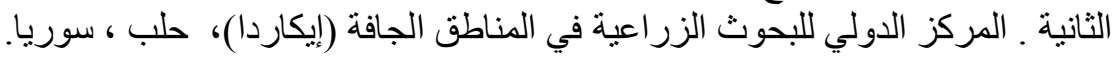

17. Pereira ,L.S. , Allen ,R.G. , and Alves , I . 1998. Irrigation water requirements .In : Persoons ,E. (ed). Functioning of hydraulic structures and monitoring of irrigation, chapter I/III of part II , Faculte des Sciences Agronomiques, Louvain -la-Neuve . Portugal .

18. Smith M., 1992. CROPWAT-A computer program for irrigation planning and management. FAO Irrigation and Drainage Paper, 46, Rome, Italy.

19. Roy ,.K.C. 2000. Irrigation scheduling of wheat based on critical growth stage method .Bangladesh J .Agril .Res .25(3):445-454.

20. النعيمي، سالم يونس.2007. الاثر الاقتصادي على للري التكميلي على منتجي القمح(دراسة مشروع الري الزي الجزيرة

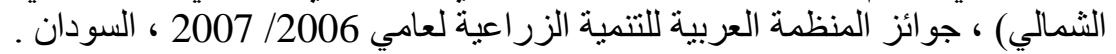

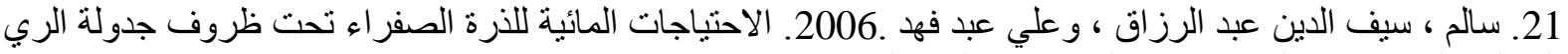

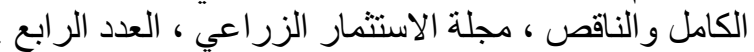

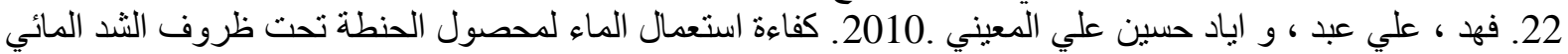

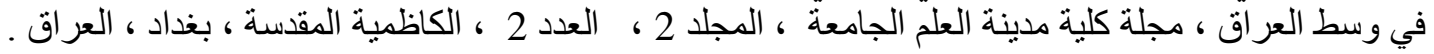
23. Adary A. , A. Hachum, T. Oweis, and M. Pala, 2002. Wheat productivity under supplemental irrigation in northern Iraq, On-Farm water research report series No.2, ICARDA, Syria .

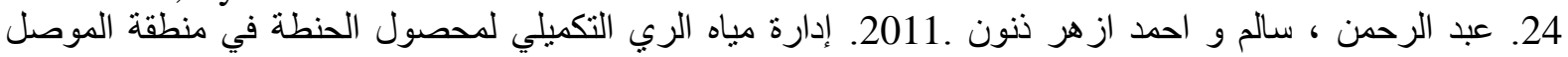

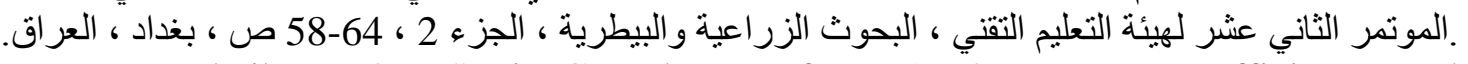

25. Zhang, H , Theib Y. O , Sonia G and Mustafa P. 1998. Water-use efficiency and transpiration efficiency of wheat under rain-fed conditions and supplemental irrigation in a Mediterranean-type environment . Plant and Soil 201: 295-305.

26. عويس ، ذيب ، الري التكميلي ، 2003. المركز الدولي للبحوث الزراعية في المناطق الجافة (إيكاردا)، حلب ،

تم اجراء البحث في المعهد التقني = ألموصل 